The Chinese Society of Theoretical and Applied Mechanics

Chinese Journal of Mechanics Press, Beijing, China

Allerton Press, INC., New York, U.S.A.

\title{
THE EFFECT OF STRAINING MODE ON VOID GROWTH*
}

\author{
Ling Xianwu (凌贤伍) Li Guochen (李国琛) \\ (LNM, Institute of Mechanics, The Chinese Academy of Sciences, Beijing 100080, China)
}

\begin{abstract}
Large strain finite element method is employed to investigate the effect of straining mode on void growth. Axisymmetric cell model embedded with spherical void is controlled by constant triaxiality loading, while plane-stress model containing a circular void is loaded by constant ratio of straining. Elastic-plastic material is used for the matrix in both cases. It is concluded that, besides the known effect of triaxiality, the straining mode which intensifies the plastic concentration around the void is also a void growth stimulator. Experimental results are cited to justify the computation results.
\end{abstract}

KEY WORDS: void growth, elastic-plastic material, damage

\section{INTRODUCTION}

The void growth in dual-phase steel sheets loaded by three paths of straining was tested by Zhang et al. ${ }^{[1]}$. Their results indicated that it was the plane-strain loading mode that yielded the fastest void growth, though its triaxiality and equivalent plastic strain were both lower than those of biaxial tension. This, however, can not be explained by the well-known models $^{[2,3]}$, so we intend to question, besides the effect of triaxiality and plastic strain, whether there is any other factor that stimulates the void growth?

Since the void growth is necessarily to be accompanied by large plastic strains around the void, the loading condition that can accelerate the plastic concentration in the matrix is well studied in this paper by using large strain finite element analysis. Two dimensional elastic-plastic cell models (axisymmetric and plane stress) are adopted. It is suggested that the nil state of the third invariant of generalized strain rate favors most the void growth. The results explain the experimental phenomenon of Zhang et al. ${ }^{[1]}$ very well and agree well with the computations of other authors.

\section{CELL MODELS AND COMPUTATION APPROACHES}

Matrix material containing periodically distributed voids can be modeled by considering a unit cell embedding single void. In Fig.1 a quadrant of this model is shown for analyzing axisymmetric $(r, z)$ and plane stress $(x, y)$ cases. The radius of the initial void is noted as $R_{0}$, the half length along the main directions of the unit cell being initially equal

Received 8 September 1998

* This paper is jointly supported by the National Natural Science Foundation of China (19872064), the Chinese Academy of Sciences (KJ951-1-201) and the Laboratory for Nonlinear Mechanics of Continuous Media of the Institute of Mechanics 


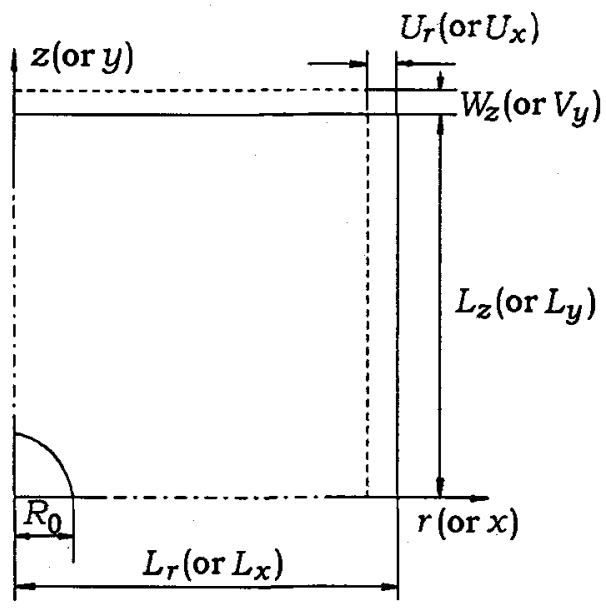

(a)

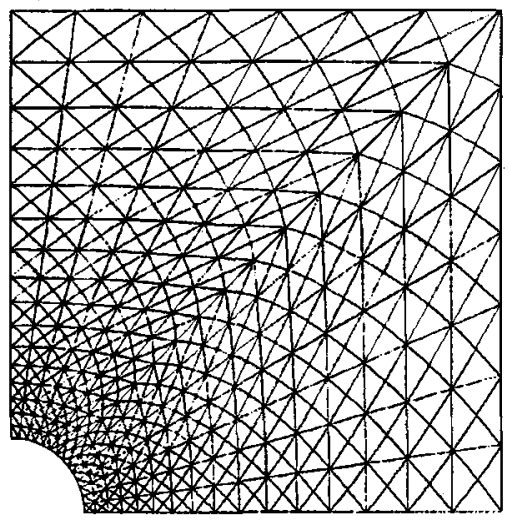

(b)

Fig.1 A quadrant of cell model and its finite element mesh

and normalized as a unit. The shape of the void is spherical in the case of axisymmetric cell, but circular throughout the thickness when plane stress model is employed.

Proportional straining is enforced on the cell whose boundaries are free from shear stress but kept straight to ensure continuity, hence the interactions between the voids are taken into account. During an incremental displacement loading, it is supposed that

$$
\left.\frac{\mathrm{d} U_{r}}{L_{r}}=\alpha \frac{\mathrm{d} W_{z}}{L_{z}} \quad \text { (i.e. } \mathrm{d} \bar{\varepsilon}_{r}=\alpha \mathrm{d} \bar{\varepsilon}_{z}\right)
$$

for the axisymmetric model, where $L_{r}$ and $U_{r}$ are respectively the current radius and the radial displacement, both normalized by the initial cell radius. Correspondingly, $L_{z}$ and $W_{z}$ are assigned as those parameters along the axial direction; and $\alpha$ is a proportional straining parameter. Similarly, we use

$$
\left.\frac{\mathrm{d} U_{x}}{L_{x}}=\alpha \frac{\mathrm{d} V_{y}}{L_{y}} \quad \text { (i.e. } \mathrm{d} \bar{\varepsilon}_{x}=\alpha \mathrm{d} \bar{\varepsilon}_{y}\right)
$$

for the plane stress model associated with similar sense in the nominations of the parameters $L_{x}, U_{x}, L_{y}$ and $V_{y}$. Here and hereafter the bar over the symbols represents the overall response of the cell so as to distinguish it from the local stress or strain.

It is assumed that the matrix material follows the incremental model of Prandtl-Reuss in plasticity with the plastic tangent modulus $E_{t e}^{(p)}$ obeying the power law. We take

$$
\frac{E_{t e}^{(p)}}{E}=n\left(\frac{\varepsilon_{\mathrm{e}}}{\varepsilon_{\mathrm{y}}}\right)^{n-1}
$$

where $E, \varepsilon_{\mathrm{e}}$ and $\varepsilon_{\mathrm{y}}$ are respectively Young's modulus, the equivalent strain and the yield strain(taken as $\varepsilon_{\mathrm{y}}=0.002$ ), and $n$ represents the exponent parameter in strain-hardening materials (given as $n=0.05,0.10$ and 0.20 ).

The method of computation is obtained with the use of the updated Lagrangian formulation for elastic-plastic large strain analysis, the theory of which has been explained in details in a previous paper ${ }^{[4]}$. The number of the incremental steps needed to complete 
each calculation varies from 500 to 3000 , depending on the ductility of the sample. The elongation along the main loading axis is selected as the generalized time $t$.

The quadrant in Fig. 1 is subdivided into 224 quadrilaterals with 4 crossed triangular elements within each of it, totaling 896 constant strain triangular elements. Computations with a much finer mesh of $1680(420 \times 4)$ elements have been employed to check the accuracy of the results and for the case when the instability of void growth is concerned. The differences of the two meshes are limited within $1 \%$ with regard to the macroscopic responses and the void growth.

\section{VOID GROWTH IN AXISYMMETRIC CELL MODEL}

During proportional straining at the boundaries of an axisymmetric cell model embedding a spherical void, the overall principal true strain $\bar{\varepsilon}_{\mathrm{r}}$ and $\bar{\varepsilon}_{z}$ can be calculated by the following relationships

$$
\bar{\varepsilon}_{z}=\ln \left(1+W_{z}\right) \quad \bar{\varepsilon}_{r}=\ln \left(1+U_{r}\right)=\alpha \bar{\varepsilon}_{z}
$$

From these we have the overall equivalent $\operatorname{strain} \bar{\varepsilon}_{\mathbf{e}}$

$$
\overline{\bar{\varepsilon}}_{\mathrm{e}}=\frac{2}{3}\left(\bar{\varepsilon}_{z}-\bar{\varepsilon}_{r}\right)
$$

The overall equivalent stress $\bar{\sigma}_{\mathrm{e}}$ and mean stress $\bar{\sigma}_{\mathrm{m}}$ can be related to the average axial stress $\bar{\sigma}_{z}$ and the average radial stress $\bar{\sigma}_{r}$ along the boundaries of the cell model as

$$
\bar{\sigma}_{\mathrm{e}}=\left|\bar{\sigma}_{z}-\bar{\sigma}_{r}\right| \quad \bar{\sigma}_{\mathrm{m}}=\frac{\bar{\sigma}_{z}+2 \bar{\sigma}_{r}}{3}
$$

The initial void volume fraction is $f_{\mathrm{v} 0}=0.225 \%$, i.e. $R_{0}=0.15$. To demonstrate the driving effects of the triaxiality $T\left(=\bar{\sigma}_{\mathrm{m}} / \bar{\sigma}_{\mathrm{e}}\right)$ and the large plastic strains around the void on the growth of void, two typical samples of $\alpha=-0.5$ and 1 are calculated with the exponent parameter $n$ being 0.2 . The contours of local equivalent stress are shown in Fig.2(a,b), when the maximum strain of a certain element within the cell reaches $\varepsilon_{\mathrm{e}}=1.0$. The strain distributions along the radial axis and the central axis are also depicted along the borders of the quadrant. In the case of $\alpha=1$, the overall equivalent strain keeps to be zero, while extremely high is the triaxiality $T(=25.98)$, plastic area covers the void as a spherical layer leaving large zone of elasticity in the outer region of the matrix, the ratio of the current void fraction to the initial one $f_{\mathrm{v}} / f_{\mathrm{v} 0}=4.28$. While in the other typical case of $\alpha=-0.5$, large plastic strain $\left(\bar{\varepsilon}_{\mathrm{e}}=0.44\right)$ is yielded with almost no void growth, that is $f_{\mathrm{v}} / f_{\mathrm{v} 0} \approx 0$, as the triaxiality keeps to be zero, although the internal matrix is covered thoroughly by large plastic strains. It is concluded that the triaxiality is the driving force of void growth: no triaxiality, no void growth; on the other hand, the large plastic strain around the void is the necessary environmental condition for driving void growth.

To demonstrate the effect of straining mode on the void growth, the constant $T$ modeling is investigated similar to those used by Koplik \& Needleman ${ }^{\left[{ }^{[}\right]}$and Brocks et al..$^{[6]}$. As has been shown by Brocks ${ }^{[6]}$, the calculation is strongly influenced by the finite element formulations under the lower triaxiality condition $(T=1)$, we therefore only choose $T=2$ and 3 . Since the material in the matrix of the cell is expected to undergo larger plastic 


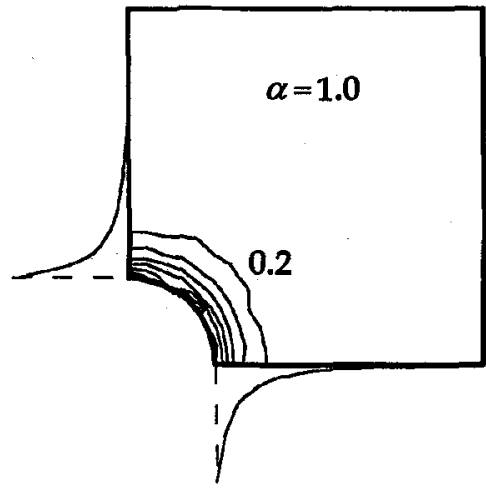

(a) $\bar{\varepsilon}_{\mathrm{e}}=0(\alpha=1)$

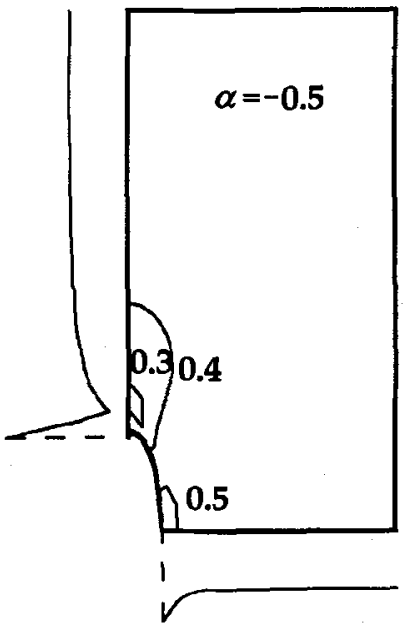

(b) $\bar{\varepsilon}_{\mathrm{e}}=0.440(\alpha=-0.5)$

Fig. 2 The contours of constant local strain $\varepsilon_{\mathrm{e}}$ (with 0.1 grading) in the axisymmetric cells $(n=0.20)$, when $\left(\varepsilon_{\mathrm{e}}\right)_{\max }=1$

strains, we have to use finer mesh for the computations (i.e. 1680 constant strain triangular elements) to avoid deterioration that would be caused by those extremely distorted elements during numerical calculations, and to extend our calculations to the point where void growth instability takes place.

In order to keep loading triaxiality constant, the proportional parameter $\alpha$ is selfadjusted in such a manner as shown in Fig.3(a) for $n=0.2$. When $\alpha$ is positive, it shows that the lateral boundary of the cell is expanding; while in negative case, it shrinks. In Fig.3(b) is shown the variation of the void fraction $f_{\mathrm{v}}$ with respect to the overall equivalent strain $\bar{\varepsilon}_{\mathrm{e}}$. At stage of $\alpha=0$ (marked as solid circle in the figures), the void growth abruptly

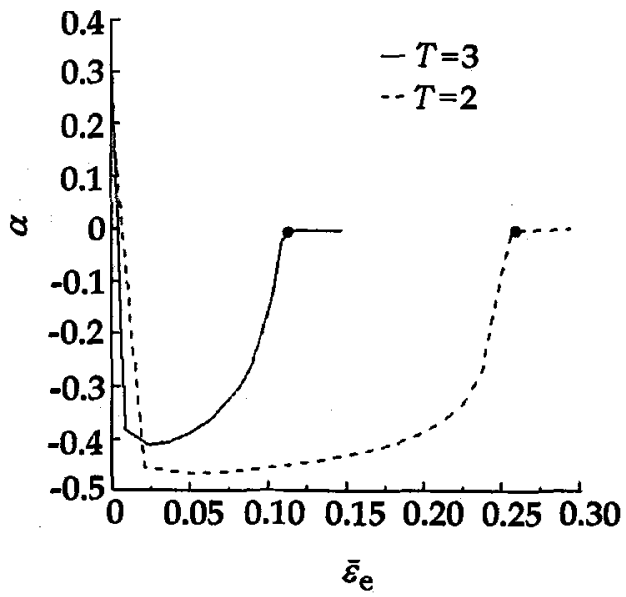

(a) The variation of the proportional straining parameter $\alpha$ with respect to overall equivalent strain $\bar{\varepsilon}_{\mathrm{e}}(n=0.20)$

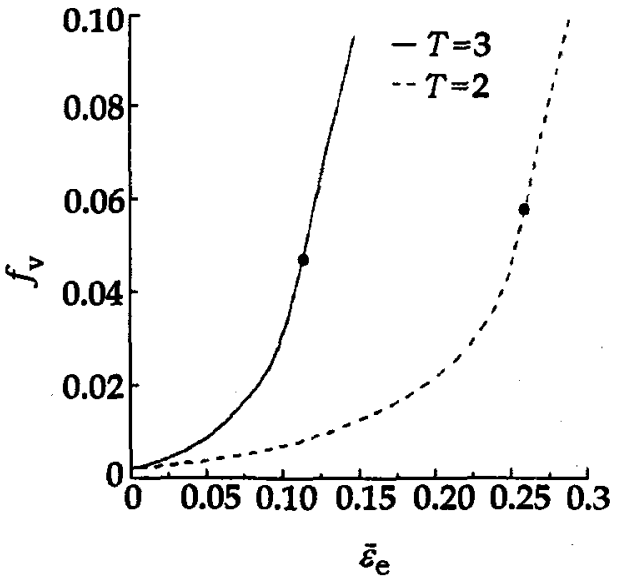

(b) The growth of void volume fraction $f_{\mathrm{v}}$ with respect to overall equivalent strain $\bar{\varepsilon}_{\mathrm{e}}$ in axisymmetric cells loaded by constant triaxiality $T=2$ and $3(n=0.20)$ 
speeds up, hence the instability occurs. The corresponding distributions of local equivalent strains at this point $\left(T=2, \bar{\varepsilon}_{\mathrm{e}}=0.260 ; T=3, \bar{\varepsilon}_{\mathrm{e}}=0.114\right)$ are given in Fig.4, when large strain concentration occurs around the void, strain localizes in the ligament between neighboring voids. The critical void volume fractions $f_{\mathrm{vc}}$, when $\alpha$ approaches zero, are similar to those given by Koplik \& Needleman ${ }^{[5]}$. The value of $f_{\mathrm{vc}}$ of $T=2$ is lower than. that of $T=3$.

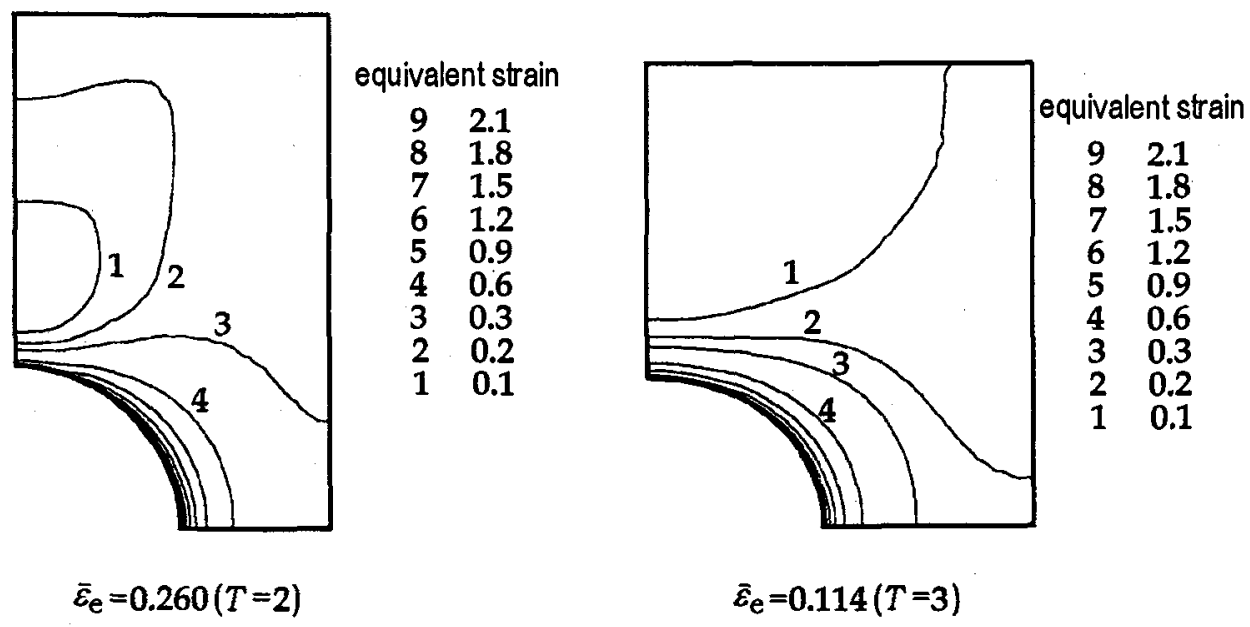

Fig.4 The contours of constant local strain $\varepsilon_{\mathrm{e}}$ in the axisymmetric cells $(n=0.20)$ loaded by constant triaxiality $T=2\left(\bar{\varepsilon}_{e}=0.260\right)$ and $T=3\left(\bar{\varepsilon}_{e}=0.114\right)$ at the instability of void growth

Since the triaxiality is kept constant, and the overall equivalent strain varies slightly under the critical state, there must exist another loading parameter that induces the abrupt growth of the void. The solution is to make use of the expression for the generalized strain rate, that is

$$
\dot{\bar{\varepsilon}}_{1} \dot{\bar{\varepsilon}}_{2} \dot{\bar{\varepsilon}}_{3}=0
$$

where 1, 2 and 3 are referring to the three main axes of strain rate in continuum. Equation (7) implies that if any main strain rate turns to zero (except the meaningless case of having three of them equal to zero), it would be a dangerous condition of inducing rapid void growth. We may refer to this criterion as the vanishing condition of the third invariant of generalized strain rate (the rate is counted on the change with respect to the generalized time). We will see in the next section that criterion (7) can also be applied to plane stress modeling of void growth.

\section{VOID GROWTH IN PLANE STRESS CELL MODEL}

In the case of plane stress cell model, the normal mesh (896 constant strain triangular elements) is adopted with all the computations terminated at the point when maximum local strain $\varepsilon_{\mathrm{e}}=1$ is reached locally in the matrix. The overall equivalent strain in its integration form is

$$
\bar{\varepsilon}_{\mathrm{e}}=\int_{0}^{t} \mathrm{~d} \bar{\varepsilon}_{\mathrm{e}}
$$


and

$$
\mathrm{d} \tilde{\varepsilon}_{\mathrm{e}}=\frac{\sqrt{2}}{3}\left[\left(\mathrm{~d} \bar{\varepsilon}_{x}-\mathrm{d} \bar{\varepsilon}_{y}\right)^{2}+\left(\mathrm{d} \bar{\varepsilon}_{y}-\mathrm{d} \bar{\varepsilon}_{z}\right)^{2}+\left(\mathrm{d} \bar{\varepsilon}_{z}-\mathrm{d} \bar{\varepsilon}_{x}\right)^{2}\right]^{1 / 2}
$$

In Eqs.(8), $\mathrm{d} \bar{\varepsilon}_{x}$ and $\mathrm{d} \bar{\varepsilon}_{y}$ are the incremental loading strains given by Eq.(2), $\mathrm{d} \bar{\varepsilon}_{z}$ is the incremental strain compressed along the thickness and counted as an average over the whole areal plane of the matrix. The overall equivalent stress $\bar{\sigma}_{\mathrm{e}}$ and the mean stress $\bar{\sigma}_{\mathrm{m}}$ can be calculated by using

$$
\bar{\sigma}_{\mathrm{e}}=\left(\bar{\sigma}_{x}^{2}-\bar{\sigma}_{x} \bar{\sigma}_{y}+\bar{\sigma}_{y}^{2}\right)^{1 / 2} \quad \bar{\sigma}_{\mathrm{m}}=\frac{\bar{\sigma}_{x}+\bar{\sigma}_{y}}{3}
$$

where $\bar{\sigma}_{x}$ and $\bar{\sigma}_{y}$ are the average stresses along the boundary sections.

To check the validity of this two dimensional plane stress model, we calculated the pure shear condition $(\alpha=-1)$. The distributions of the local equivalent strain in the cell are very similar to those given by Hom $\&$ McMeeking ${ }^{[7]}$ at the middle cross section of their three dimensional cell model. Under the shearing condition, void radius also enlarges along the axis of tension and shrinks along the axis of compression with negligible void volumetric change.

In Fig.5(a) are shown the relationship between the void volume fraction $f_{\mathrm{v}}$ and the overall equivalent strain $\bar{\varepsilon}_{\mathrm{e}}$ for $\alpha=1,0$ and -0.45 with the strain hardening exponent $n=0.05,0.10$ and 0.20 . The corresponding conditions of the triaxiality $T=\bar{\sigma}_{\mathrm{m}} / \bar{\sigma}_{\mathrm{e}}$ are given in Fig.5(b). It is found that it is under the plane strain mode of loading $(\alpha=0)$ that the void grows the fastest, even faster than the biaxial case $(\alpha=1.0)$, although its triaxiality level, and also its overall equivalent strain, are lower. Another important feature is that the void grows faster if the matrix is softer ( $\operatorname{smaller} n$ ) even if the triaxiality is lower. To explain this paradoxical phenomenon, let us define a strain concentration factor as

$$
\varepsilon_{\mathrm{s}}=\left(\varepsilon_{\mathrm{e}}\right)_{\max } / \bar{\varepsilon}_{\mathrm{e}}
$$

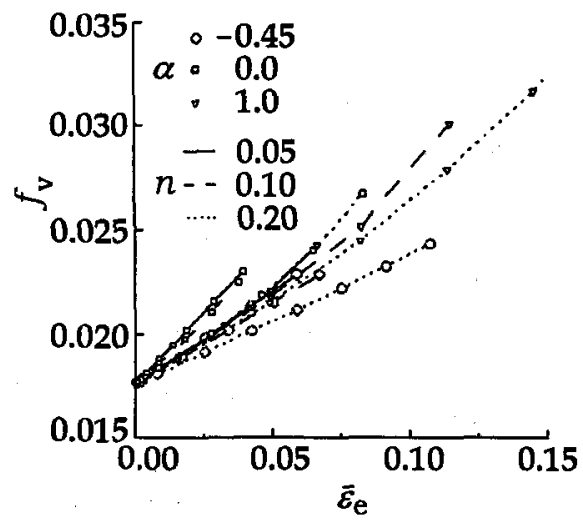

(a) The growth of void volume fraction $f_{\mathrm{v}}$ with respect to overall equivalent strain $\bar{\varepsilon}_{\mathrm{e}}$

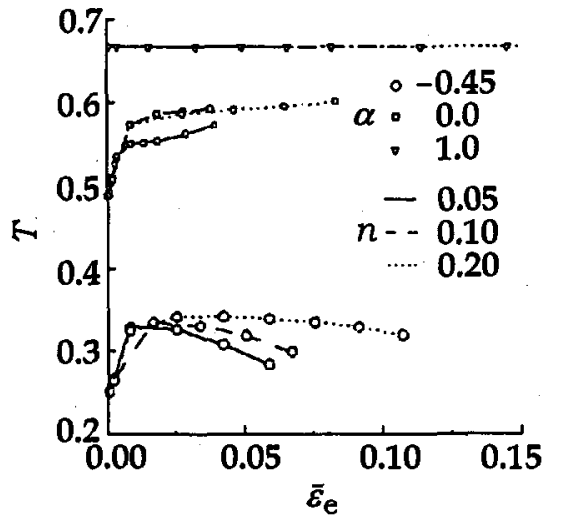

(b) The relationship between the triaxiality parameter $T$ and overall equivalent strain $\bar{\varepsilon}_{\mathrm{e}}$ in plane stress cells with various strain hardening exponents $n=0.05,0.10,0.20$ 
which is the ratio between the maximum value of the local equivalent strain $\varepsilon_{\mathrm{e}}$ in the matrix and the overall equivalent strain $\bar{\varepsilon}_{\mathrm{e}}$ loaded on the cell. Table 1 gives a list of the values of the strain concentration factor $\varepsilon_{\mathrm{s}}$ when all the calculations stops at the same maximum local strain $\varepsilon_{\mathrm{e}}=1$. In all cases, the straining condition of $\alpha=0$ yields the largest value of $\varepsilon_{s}$ and the softer the matrix (smaller $n$ ), the larger the value of strain concentration factor. As can be seen from Fig.5(b), the difference of triaxiality between the two cases of $\alpha=0$ and $\alpha=1$ is not large, it should then be the influence of large plastic flow around the void that becomes overwhelming. Hence straining mode $(\alpha=0)$ comes into effect through its influence on the internal strain distribution around the void.

Table 1 Strain concentration factor $\varepsilon_{\mathrm{s}}$ when local equivalent strain $\varepsilon_{\mathrm{e}}=1$

\begin{tabular}{cccc}
\hline & \multicolumn{3}{c}{$n$} \\
\cline { 2 - 4 }$\alpha$ & 0.05 & 0.1 & 0.2 \\
\hline-0.45 & 16.92 & 13.17 & 8.32 \\
0 & 23.87 & 19.46 & 11.26 \\
1 & 9.86 & 7.43 & 4.96 \\
\hline
\end{tabular}

Concentration of large plastic strain not only plays the role as an adjoint factor of voiding but also acts as the stimulator of its growth, since large equivalent strain lowers down the plastic stiffness around the void, as is seen from Eq.(3). The concentration of large strains around the void further influences the distribution of strain energy within the matrix material. Assume an inner layer as $A$ around the void with its exterior radius as $2 R_{0}$, the remaining part of the matrix as $B$, we have the strain energy ratio of the inner part $A$ as

$$
\rho_{\mathrm{se}}=\int_{0}^{t} \int_{A} \sigma_{i j} D_{i j} \mathrm{~d} s \mathrm{~d} t / \int_{0}^{t} \int_{A+B} \sigma_{i j} D_{i j} \mathrm{~d} s \mathrm{~d} t
$$

where $\sigma_{i j}$ is the true stress within the matrix, $D_{i j}=\frac{1}{2}\left(V_{i, j}+V_{j, i}\right)$ is the deformation rate comprised of the velocity $V_{i}, s$ is the area of the matrix. The variations of $\rho_{\mathrm{se}}$ with $\bar{\varepsilon}_{\mathrm{e}}$ are depicted in Fig.6 for the three loading conditions $(\alpha=1,0,-0.45)$. It is indicated that the case of plane strain loading $(\alpha=0)$ has the largest proportion of strain energy for its inner area. Also, the softer the matrix (lower value of $n$ ), the higher is the proportion of $\rho_{\text {se. }}$.

These examples demonstrate that the overall straining mode, characterized by criterion (7), influences not only the local strain concentration around the void but also the distribution of strain energy within the matrix. More energy is stored/dissipated in the

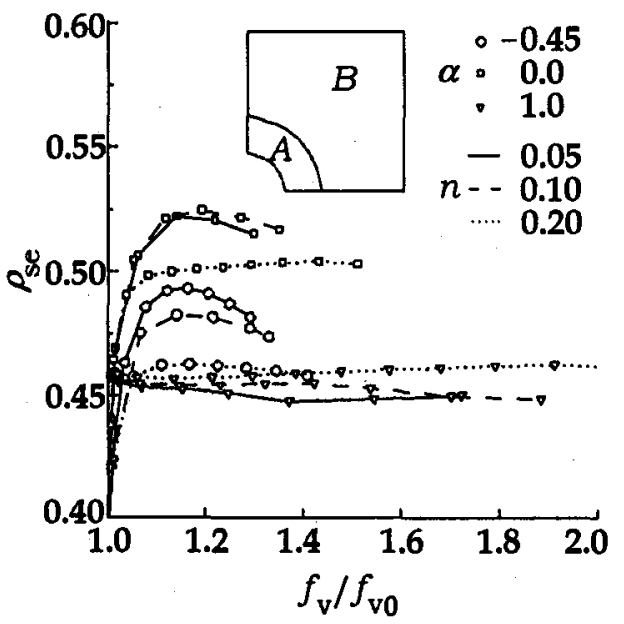

Fig.6 The variation of the strain energy ratio $\rho_{\mathrm{se}}$ with respect to the void growth parameter $f_{\mathrm{v}} / f_{\mathrm{v} 0}$ (the exterior radius of region $A$ is $2 R_{0}$ )

adjoining area of the void when the criterion is reached, thus incurring much faster void growth. The hardness of the matrix material (characterized by the parameter $n$ ) would also exert an influence on the assignment of this proportion. 
In comparison with the tests done by Zhang et al. ${ }^{[1]}$, we can now explain the phenomenon which can not be explained by the other models ${ }^{[2,3]}$.

Based on a simple form of the deformation type of plastic equations for continuum, that is

$$
\bar{\varepsilon}_{i j}=\Psi \bar{S}_{i j}
$$

where $\Psi=3 \bar{\varepsilon}_{\mathrm{e}} / 2 \bar{\sigma}_{\mathrm{e}}$, the triaxiality parameter under constant strain ratio $\left(\alpha=\varepsilon_{2} / \varepsilon_{1}\right)$ loading can be calculated by the formula

$$
T=\bar{\sigma}_{\mathrm{m}} / \bar{\sigma}_{\mathrm{e}}=\frac{1+\alpha}{\sqrt{3}\left(1+\alpha+\alpha^{2}\right)^{1 / 2}}
$$

In their experiments for dual-phase steel sheets, Zhang et al.[1] had $T=0.666$ for the biaxial stretching ( $\alpha=0.87$ ), 0.592 for the plane strain loading $(\alpha=0.055)$ and 0.366 for the uniaxial tension $(\alpha=-0.45)$. In our computations, we then have $T=0.667(\alpha=$ 1), $0.577(\alpha=0)$ and $0.366(\alpha=-0.45)$, which are in good agreement with the maximum values of the triaxiality results given in Fig.5(b) for all the cases.

It was found that the plane strain loading yielded larger void size along both the rolling direction and the thickness direction than those of the biaxial stretching, though its triaxiality is less than that of the latter case. Since it was the average void size, not the void areal fraction, that they measured, thus they excluded the influence of void nucleation. This trend is reproduced in our simulations, as is shown in Fig.5(a), it is the plane strain loading that incurs the fastest void growth.

\section{CONCLUSIONS}

The main conclusions of this paper can be given as follows:

(1) Void must be surrounded by large plastic strains so as to move its boundary. Therefore, besides the effect of triaxiality, the straining mode that facilitates the strain concentration around the void is also a stimulator of the void growth.

(2) The straining mode characterized as the vanishing state of the third invariant of generalized strain rate is the most deteriorating case.

(3) The straining mode comes into effect through concentrating the strains around the void, enhancing the strain energy stored/dissipated in the adjoining layer, and reducing the plastic stiffness of the matrix material near to the void.

\section{REFERENCES}

1 Zhang YZ, Hu XY, Huang JQ. Damage in sheet forming of a dual-phase steel. Res Mechanica, 1990, 31: 35 48

2 Rice JR, Tracy DM. On ductile enlargement of voids in triaxial stress fields. J Mech Phys Solids, 1969, 27: 201 217

3 Gurson AL. Continuum theory of ductile rupture by void nucleation and growth: Part I-Yield criteria and flow rule for porous ductile media. J Engng Mater Tech, 1977, 99: 2 15

$4 \mathrm{Li} \mathrm{GC}$, Howard IC. The effect of strain softening in the matrix material during void growth. $J$ Mech Phys Solids, 1983, 31: 85 102

5 Koplik J, Needleman A. Void growth and coalescence in porous plastic solids. Int $J$ Solids Structures, 1988, 24: 835 853

6 Brocks W, Sun DZ, Honig A. Verifications of the transferability of the micromechanical parameters by cell model calculations with visco-plastic materials. Int $J$ Plasticity, 1995, 11: 971 989

7 Hom CL, McMeeking RM. Void growth in elastic-plastic materials. $J A M, 1989,56:$ 309 317 